\title{
Tunneling through Intragroup Transactions: Evidence from Korean Chaebols
}

\author{
Ji Suk Lee ${ }^{1}$ and Shan Yue Jin ${ }^{2}$ \\ ${ }^{1,2}$ Korea Institute of Industrial Technology Gachon University, Korea \\ 1jslee82@kitech.re.kr, ${ }^{2}$ jsyrena0923@gachon.ac.kr
}

\begin{abstract}
This study examines the interaction between the ownership structure and the intragroup transactions of Korean chaebols to test the tunneling hypothesis. Using the data on intragroup transactions (sales, purchases, receivables, and payables) and intragroup ownership between 1999 and 2014, we derive two findings. First, the intragroup transactions of firms with large and/or direct ownership by the controlling family enhance the firms' performance significantly more than those of firms with small and/or indirect ownership by the family. Second, firms' performance improves more if the counterparties of the intragroup transactions are firms with smaller and/or more indirect ownership by family and also with counterparty firms that are central to the group. The results are consistent with the tunneling hypothesis and suggest that intragroup transactions are important means of tunneling activities by controlling families.
\end{abstract}

Keywords: Intragroup transactions, Tunneling, family business groups, Ownership structure, Controlling family

\section{Introduction}

Many firms worldwide are controlled by families. Controlling families typically exert power over their firms significantly over their cash flow rights, primarily through pyramidal or crossholding ownership structures and dual-class shares.

The empirical literature has focused primarily on the relationship between the cash flow and voting rights of the controlling families and the performance and value of their firms [2][3][5][6][7][8]. Meanwhile, some studies use ownership as an endogenous variable and investigate firm profitability as a factor that affects ownership structure [4].

Although [1] set their selection hypothesis against the tunneling argument, their findings do not indicate that tunneling does not happen in family business groups; therefore, they do not preclude a possibility that tunneling may happen after determining the group firms' ownership structure to affect firm profitability. In addition, their tunneling tests only focus on the transfer of wealth from pyramid firms to the firms owned by controlling families. They do not examine comprehensive tunneling that could occur within family business groups.

How, then, do tunneling activities occur to increase the controlling family's wealth? If tunneling occurs in family business groups, the main and direct channel must be intragroup transactions such as sales and purchases of goods and services. However, despite the considerable anecdotal evidence, little evidence is available regarding the transactions through which expropriation occurs.

Article history:

Received (February 14, 2019), Review Result (March 10, 2019), Accepted (April 14, 2019) 
This paper contributes to the literature on family business groups in several ways. First, it provides direct evidence for the tunneling hypothesis by showing that firms closely related to the controlling family gain more through intragroup transactions than other group firms. Second, this paper examines counterparties' characteristics to determine if the recipients gain through any of the transaction channels. The results shed new light on the direct channels of tunneling through intragroup transactions. Third, the hypotheses are tested using comprehensive data on ownership and intragroup transactions that are not generally available in most countries. Finally, additional tests are performed to support the arguments, and detailed and clear explanations of the tunneling activities pursued by controlling families are provided.

\section{Hypotheses}

H1. Companies with a large share of family ownership gain from intragroup transactions whereas companies with a small share of family ownership lose from intragroup transactions.

H2. Companies with direct family ownership gain from intragroup transactions whereas companies with indirect family ownership lose from intragroup transactions.

H3. Companies with large or direct family ownership gain from their transactions with companies with small or indirect family ownership.

H4. Companies with a large or direct family ownership gain from their transactions with central companies.

\section{Regression models and variables}

To investigate hypotheses 1 to 4 , the following model is used to test whether the impact of intragroup transactions on firm performance differs according to the firm's ownership structure.

$$
\begin{aligned}
{\text { Profit }=\beta_{0}+}_{+} & \beta_{1} \text { Transaction }_{i t}+\beta_{2} \text { Ownership }_{i t}+\beta_{3} \text { Ownership dum }_{i t} \\
& * \text { Transaction }_{i t}+\Phi \text { Controls }_{i t-1}+P D+Y D+I D+G D \\
& +\varepsilon_{i t}
\end{aligned}
$$

Profit: Net income / Total assets

Transaction:

(1) Sales = Intragroup sales / Total sales

(2) Purchases $=$ Intragroup purchases $/$ Total purchases

(3) Receivables $=$ Intragroup receivables $/$ Total receivables

(4) Payables $=$ Intragroup payables / Total payables

Ownership: (1) Ultimate ownership (2) Position

Ownership dum: (1) Large (2) Direct

Controls: Firm size \& Leverage

PD (Public dummy), YD (Year dummies), ID (Industry dummies), GD (Group dummies)

$\varepsilon$ : Error term 


\section{Empirical results}

[Table 1] and [Table 2] test for H. 1 and 2. The dependent variable is profitability and it is defined as net income divided by total assets. Sales is the intragroup sales / total sales and Purchases is intragroup purchases / total purchases (cost of sales + selling \& administrative expense), Receivables is intragroup receivables / total receivables (accounted receivable + investment assets + other current or non-current receivables) and Payables is intragroup payables / total payables (total liabilities). Ownership variables are ultimate ownership, ultimate cash flow rights of family, and position, the distance between the family and a firm in the group. Large indicates the firms whose ultimate ownership is more than 0.3 and direct indicates the firms whose position is less than 2. Firm size is the logarithm of the book value of total assets, leverage is defined as total liabilities divided by assets and public dummy is a variable that takes the value of one of the firms is listed. T-statistics are in parenthesis.

Table 1. The results of empirical analysis on the effects of large or direct ownership (sales) on profitability

\begin{tabular}{|c|c|c|c|c|}
\hline & \multicolumn{4}{|c|}{ Dependent variable: Profitability } \\
\hline & (1) & (2) & (3) & (4) \\
\hline Sales & $\begin{array}{l}-0.005 \\
(-1.17)\end{array}$ & $\begin{array}{c}-0.011 * * \\
(-1.98)\end{array}$ & $\begin{array}{l}-0.006 \\
(-1.25)\end{array}$ & $\begin{array}{c}-0.014 * * * \\
(-2.65)\end{array}$ \\
\hline Purchases & $\begin{array}{l}-0.008 \\
(-1.33) \\
\end{array}$ & $\begin{array}{l}-0.005 \\
(-0.76) \\
\end{array}$ & $\begin{array}{l}-0.007 \\
(-1.17) \\
\end{array}$ & $\begin{array}{l}-0.006 \\
(-0.96) \\
\end{array}$ \\
\hline Ultimate ownership & $\begin{array}{c}0.046^{* * * *} \\
(6.62) \\
\end{array}$ & $\begin{array}{c}0.040 * * * \\
(4.96) \\
\end{array}$ & & \\
\hline Position & & & $\begin{array}{c}-0.012 * * * \\
(-6.38)\end{array}$ & $\begin{array}{c}-0.008 * * * \\
(-3.87)\end{array}$ \\
\hline Large * Sale & & $\begin{array}{c}0.015^{*} \\
(1.85)\end{array}$ & & \\
\hline Large * Purchase & & $\begin{array}{l}-0.006 \\
(-0.51) \\
\end{array}$ & & \\
\hline Direct $*$ Sale & & & & $\begin{array}{c}0.029 * * * \\
(3.36)\end{array}$ \\
\hline Direct $*$ Purchase & & & & $\begin{array}{l}0.001 \\
(0.08) \\
\end{array}$ \\
\hline Firm size & $\begin{array}{l}0.001 \\
(1.00) \\
\end{array}$ & $\begin{array}{l}0.001 \\
(0.92) \\
\end{array}$ & $\begin{array}{l}-0.000 \\
(-0.31) \\
\end{array}$ & $\begin{array}{l}-0.000 \\
(-0.30) \\
\end{array}$ \\
\hline Leverage & $\begin{array}{c}-0.116^{* * * *} \\
(-21.28)\end{array}$ & $\begin{array}{c}-0.116^{* * *} \\
(-21.28)\end{array}$ & $\begin{array}{c}-0.114 * * * \\
(-20.96)\end{array}$ & $\begin{array}{c}-0.114 * * * \\
(-21.00)\end{array}$ \\
\hline Public dummy & $\begin{array}{l}-0.007 \\
(-1.57) \\
\end{array}$ & $\begin{array}{l}-0.007 \\
(-1.57) \\
\end{array}$ & $\begin{array}{c}-0.011 * * * \\
(-2.59) \\
\end{array}$ & $\begin{array}{c}-0.011 * * \\
(-2.52) \\
\end{array}$ \\
\hline Constant & $\begin{array}{l}-0.011 \\
(-0.35) \\
\end{array}$ & $\begin{array}{l}-0.007 \\
(-0.22) \\
\end{array}$ & $\begin{array}{c}0.069 * * \\
(2.06) \\
\end{array}$ & $\begin{array}{c}0.064 * \\
(1.91) \\
\end{array}$ \\
\hline YD, ID, GD & Yes & Yes & Yes & Yes \\
\hline Observations & 6,748 & 6,748 & 6,748 & 6,748 \\
\hline F Value & 15.55 & 15.22 & 15.51 & 15.29 \\
\hline Adj R-Sq & 0.15 & 0.15 & 0.15 & 0.15 \\
\hline
\end{tabular}


Table 2. The results of empirical analysis on the effects of ownership (purchases) on profitability

\begin{tabular}{|c|c|c|c|c|}
\hline & \multicolumn{4}{|c|}{ Dependent variable: Profitability } \\
\hline & (1) & (2) & (3) & (4) \\
\hline Receivables & $\begin{array}{l}0.005 \\
(1.37)\end{array}$ & $\begin{array}{l}-0.002 \\
(-0.52)\end{array}$ & $\begin{array}{l}0.005 \\
(1.39)\end{array}$ & $\begin{array}{l}0.004 \\
(0.89)\end{array}$ \\
\hline Payables & $\begin{array}{c}-0.013 * \\
(-1.89)\end{array}$ & $\begin{array}{c}0.007 \\
(0.083)\end{array}$ & $\begin{array}{c}-0.013 * \\
(-1.90)\end{array}$ & $\begin{array}{l}-0.012 \\
(-1.53)\end{array}$ \\
\hline Ultimate ownership & $\begin{array}{c}0.034 * * * \\
(4.42)\end{array}$ & $\begin{array}{c}0.034 * * * \\
(3.98)\end{array}$ & & \\
\hline Position & & & $\begin{array}{c}-0.011 * * * \\
(-5.44)\end{array}$ & $\begin{array}{c}-0.010 * * * \\
(-4.39)\end{array}$ \\
\hline Large * Receivables & & $\begin{array}{c}0.024 * * * \\
(3.27)\end{array}$ & & \\
\hline Large * Payables & & $\begin{array}{l}-0.005 \\
(-0.43)\end{array}$ & & \\
\hline Direct * Receivables & & & & $\begin{array}{c}0.015^{*} \\
(1.68)\end{array}$ \\
\hline Direct * Payables & & & & $\begin{array}{l}-0.005 \\
(-0.34)\end{array}$ \\
\hline Firm size & $\begin{array}{l}0.001 \\
(0.56)\end{array}$ & $\begin{array}{l}0.000 \\
(0.41)\end{array}$ & $\begin{array}{l}-0.001 \\
(-0.50)\end{array}$ & $\begin{array}{l}-0.001 \\
(-0.45)\end{array}$ \\
\hline Leverage & $\begin{array}{c}-0.130 * * * \\
(-21.20)\end{array}$ & $\begin{array}{c}-0.129 * * * \\
(-21.05)\end{array}$ & $\begin{array}{c}-0.129 * * * \\
(-21.09)\end{array}$ & $\begin{array}{c}-0.129 * * * \\
(-21.04)\end{array}$ \\
\hline Public dummy & $\begin{array}{c}-0.010 * * \\
(-2.15)\end{array}$ & $\begin{array}{c}-0.009 * * \\
(-2.02)\end{array}$ & $\begin{array}{c}-0.013 * * * \\
(-2.88)\end{array}$ & $\begin{array}{c}-0.013 * * \\
(-2.82)\end{array}$ \\
\hline Constant & $\begin{array}{l}0.046 \\
(1.18) \\
\end{array}$ & $\begin{array}{l}0.047 \\
(1.20) \\
\end{array}$ & $\begin{array}{c}0.117 * * * \\
(2.06)\end{array}$ & $\begin{array}{c}0.113 * * * \\
(2.80)\end{array}$ \\
\hline YD, ID, GD & Yes & Yes & Yes & Yes \\
\hline Observations & 5,059 & 5,059 & 5,059 & 5,059 \\
\hline F Value & 14.70 & 14.70 & 14.86 & 14.50 \\
\hline Adj R-Sq & 0.17 & 0.18 & 0.17 & 0.17 \\
\hline
\end{tabular}

[Table 3] and [Table 4] tests for H. 3 and 4 with intragroup sales. The dependent variable is profitability and it is defined as net income divided by total assets. Sales are dependent on intragroup sales and measured by the intragroup sales / total sales, purchases (Purs) are dependent on intragroup purchase and measured by intragroup purchases / total purchases (cost of sales + selling $\&$ administrative expense). $\mathrm{L}$ is the set of the counterparties with low ultimate ownership ( $\mathrm{UO}<0.3)$, low position $(\mathrm{POS}<2)$, or low centrality $(\mathrm{CEN}=0)$. $\mathrm{H}$ is the set of the counterparties with high ultimate ownership $(\mathrm{UO}>=0.3)$, high position $(\mathrm{POS}>=2)$, or high centrality $(\mathrm{CEN}>0)$. Ultimate ownership is the ultimate cash flow right off the family and position is the distance between the family and a firm in the group. OD (Ownership dummy) indicates the firms whose ultimate ownership is more than 0.3 or position is less than 2. Firm size is the logarithm of the book value of total assets, leverage is defined as total liabilities divided by assets and public dummy is a variable that takes the value of one of the firms is listed. T-statistics are in parenthesis. 
Table 3. The results of empirical analysis on the effects of ultimate ownership and position (sales) on profitability

\begin{tabular}{|c|c|c|c|c|c|c|c|c|}
\hline & \multicolumn{8}{|c|}{ Dependent variable: Profitability } \\
\hline & (1) & (2) & (3) & (4) & $(5)$ & (6) & (7) & (8) \\
\hline Criteria for $\mathrm{L} / \mathrm{H}$ & & $\mathrm{UO}$ & POS & CEN & & $\mathrm{UO}$ & POS & CEN \\
\hline $\begin{array}{c}\text { Ownership } \\
\text { dummy(OD) }\end{array}$ & \multicolumn{4}{|c|}{ Large ownership dummy } & \multicolumn{4}{|c|}{ Direct ownership dummy } \\
\hline Sales & $\begin{array}{c}-0.010^{*} \\
(-1.92)\end{array}$ & & & & $\begin{array}{c}-0.014 * * * \\
(-2.63)\end{array}$ & & & \\
\hline Sales to $\mathrm{L}$ & & $\begin{array}{c}-0.013 * * \\
(-2.29) \\
\end{array}$ & $\begin{array}{l}-0.004 \\
(-0.63) \\
\end{array}$ & $\begin{array}{l}-0.026 \\
(-1.56) \\
\end{array}$ & & $\begin{array}{c}-0.015^{* *} \\
(-2.52) \\
\end{array}$ & $\begin{array}{l}-0.005 \\
(-0.80) \\
\end{array}$ & $\begin{array}{c}-0.047 * * * \\
(-3.64) \\
\end{array}$ \\
\hline Sales to $\mathrm{H}$ & & $\begin{array}{l}0.024 \\
(1.47) \\
\end{array}$ & $\begin{array}{c}-0.016^{* *} \\
(-2.22) \\
\end{array}$ & $\begin{array}{l}-0.008 \\
(-1.41) \\
\end{array}$ & & $\begin{array}{l}-0.008 \\
(-0.78) \\
\end{array}$ & $\begin{array}{c}-0.022 * * * \\
(-3.08)\end{array}$ & $\begin{array}{l}-0.007 \\
(-1.31) \\
\end{array}$ \\
\hline $\begin{array}{c}\text { Ultimate } \\
\text { ownership }\end{array}$ & $\begin{array}{c}0.038 * * * \\
(4.94) \\
\end{array}$ & $\begin{array}{c}0.037 * * * \\
(4.74) \\
\end{array}$ & $\begin{array}{c}0.039 * * * \\
(4.99) \\
\end{array}$ & $\begin{array}{c}0.039 * * * \\
(4.99) \\
\end{array}$ & & & & \\
\hline Position & & & & & $\begin{array}{c}-0.008 * * * \\
(-4.01) \\
\end{array}$ & $\begin{array}{c}-0.009 * * * \\
(-4.12) \\
\end{array}$ & $\begin{array}{c}-0.008 * * * \\
(-3.63) \\
\end{array}$ & $\begin{array}{c}-0.009 * * * \\
(-4.33) \\
\end{array}$ \\
\hline OD $*$ Sales & $\begin{array}{c}0.015^{*} \\
(1.77)\end{array}$ & & & & $\begin{array}{c}0.028 * * * \\
(3.34)\end{array}$ & & & \\
\hline $\mathrm{OD} *$ Sales to $\mathrm{L}$ & & $\begin{array}{c}0.032 * * * \\
(2.75)\end{array}$ & $\begin{array}{l}0.014 \\
(1.28)\end{array}$ & $\begin{array}{l}-0.011 \\
(-0.50)\end{array}$ & & $\begin{array}{c}0.027 * * \\
(2.50)\end{array}$ & $\begin{array}{l}0.013 \\
(1.22)\end{array}$ & $\begin{array}{c}0.054 * * \\
(2.28)\end{array}$ \\
\hline $\mathrm{OD} *$ Sales to $\mathrm{H}$ & & $\begin{array}{c}-0.030^{*} \\
(-1.69)\end{array}$ & $\begin{array}{l}0.020^{*} \\
(1.78)\end{array}$ & $\begin{array}{c}0.022 * * \\
(2.34)\end{array}$ & & $\begin{array}{l}0.021 \\
(1.50)\end{array}$ & $\begin{array}{c}0.050 * * * \\
(3.38)\end{array}$ & $\begin{array}{c}0.021 * * \\
(2.11)\end{array}$ \\
\hline Firm size & $\begin{array}{l}0.001 \\
(0.87) \\
\end{array}$ & $\begin{array}{l}0.001 \\
(0.89) \\
\end{array}$ & $\begin{array}{l}0.001 \\
(0.89) \\
\end{array}$ & $\begin{array}{l}0.001 \\
(0.88) \\
\end{array}$ & $\begin{array}{l}-0.001 \\
(-0.34) \\
\end{array}$ & $\begin{array}{l}-0.000 \\
(-0.34) \\
\end{array}$ & $\begin{array}{l}-0.001 \\
(-0.32) \\
\end{array}$ & $\begin{array}{l}-0.000 \\
(-0.27) \\
\end{array}$ \\
\hline Leverage & $\begin{array}{c}-0.116 * * * \\
(-21.43) \\
\end{array}$ & $\begin{array}{c}-0.117 * * * \\
(-21.52) \\
\end{array}$ & $\begin{array}{c}-0.116^{* * *} * \\
(-21.37) \\
\end{array}$ & $\begin{array}{c}-0.116^{* * * *} \\
(-21.33) \\
\end{array}$ & $\begin{array}{c}-0.115 * * * \\
(-21.14) \\
\end{array}$ & $\begin{array}{c}-0.115 * * * \\
(-21.12) \\
\end{array}$ & $\begin{array}{c}-0.115 * * * \\
(-21.18) \\
\end{array}$ & $\begin{array}{c}-0.115 * * * \\
(-21.14) \\
\end{array}$ \\
\hline Public dummy & $\begin{array}{l}-0.007 \\
(-1.60) \\
\end{array}$ & $\begin{array}{l}-0.007 \\
(-1.63) \\
\end{array}$ & $\begin{array}{l}-0.007 \\
(-1.60) \\
\end{array}$ & $\begin{array}{l}-0.007 \\
(-1.54) \\
\end{array}$ & $\begin{array}{c}-0.011 * * \\
(-2.54) \\
\end{array}$ & $\begin{array}{c}-0.011 * * * \\
(-2.56) \\
\end{array}$ & $\begin{array}{c}-0.011 * * \\
(-2.45) \\
\end{array}$ & $\begin{array}{c}-0.011 * * * \\
(-2.58) \\
\end{array}$ \\
\hline Constant & $\begin{array}{l}-0.006 \\
(-0.18) \\
\end{array}$ & $\begin{array}{l}-0.003 \\
(-0.09) \\
\end{array}$ & $\begin{array}{l}-0.006 \\
(-0.18) \\
\end{array}$ & $\begin{array}{l}-0.004 \\
(-0.11) \\
\end{array}$ & $\begin{array}{c}0.065^{*} \\
(1.95)\end{array}$ & $\begin{array}{c}0.065^{*} \\
(1.95) \\
\end{array}$ & $\begin{array}{c}0.063^{*} \\
(1.89) \\
\end{array}$ & $\begin{array}{c}0.067 * * \\
(2.00) \\
\end{array}$ \\
\hline YD, ID, GD & Yes & Yes & Yes & Yes & Yes & Yes & Yes & Yes \\
\hline Observations & 6,732 & 6,732 & 6,732 & 6,732 & 6,732 & 6,732 & 6,732 & 6,732 \\
\hline F Value & 15.74 & 15.47 & 15.39 & 15.48 & 15.82 & 15.41 & 15.48 & 15.53 \\
\hline Adj R-Sq & 0.15 & 0.15 & 0.15 & 0.15 & 0.15 & 0.15 & 0.15 & 0.15 \\
\hline
\end{tabular}

Table 4. The results of empirical analysis on the effects of ultimate ownership and position (purchases) on profitability

\begin{tabular}{|c|c|c|c|c|c|c|c|c|}
\hline & \multicolumn{8}{|c|}{ Dependent variable: Profitability } \\
\hline & (1) & (2) & (3) & (4) & (5) & (6) & (7) & (8) \\
\hline $\begin{array}{c}\text { Criteria for } \\
\mathrm{L} / \mathrm{H}\end{array}$ & & UO & POS & CEN & & UO & POS & CEN \\
\hline $\begin{array}{c}\text { Ownership } \\
\text { dummy (OD) }\end{array}$ & \multicolumn{4}{|c|}{ Large ownership dummy } & \multicolumn{4}{|c|}{ Direct ownership dummy } \\
\hline Purchases & $\begin{array}{l}-0.006 \\
(-0.91)\end{array}$ & & & & $\begin{array}{l}-0.008 \\
(-1.21) \\
\end{array}$ & & & \\
\hline Purs from $L$ & & $\begin{array}{l}-0.002 \\
(-0.26) \\
\end{array}$ & $\begin{array}{l}-0.011 \\
(-1.16) \\
\end{array}$ & $\begin{array}{l}-0.018 \\
(-0.87) \\
\end{array}$ & & $\begin{array}{c}-0.001 * * * \\
(-0.06) \\
\end{array}$ & $\begin{array}{l}-0.010 \\
(-1.12) \\
\end{array}$ & $\begin{array}{c}-0.036^{*} \\
(-1.81) \\
\end{array}$ \\
\hline Purs from $\mathrm{H}$ & & -0.019 & 0.002 & -0.003 & & $-0.024 *$ & -0.003 & -0.003 \\
\hline
\end{tabular}




\begin{tabular}{|c|c|c|c|c|c|c|c|c|}
\hline & & $(-1.12)$ & $(0.24)$ & $(-0.42)$ & & $(-1.91)$ & $(-0.26)$ & $(-0.43)$ \\
\hline $\begin{array}{c}\text { Ultimate } \\
\text { ownership }\end{array}$ & $\begin{array}{c}0.045^{* * *} * \\
(6.25)\end{array}$ & $\begin{array}{c}0.044 * * * \\
(6.21)\end{array}$ & $\begin{array}{c}0.045^{* * *} * \\
(6.35)\end{array}$ & $\begin{array}{c}0.046 * * * \\
(6.41)\end{array}$ & & & & \\
\hline Position & & & & & $\begin{array}{c}-0.011 * * * \\
(-5.81)\end{array}$ & $\begin{array}{c}-0.011 * * * \\
(-5.86)\end{array}$ & $\begin{array}{c}-0.012 * * * \\
(-5.89)\end{array}$ & $\begin{array}{c}-0.011 * * * \\
(-5.86)\end{array}$ \\
\hline $\begin{array}{c}\text { OD * } \\
\text { Purchases }\end{array}$ & $\begin{array}{l}-0.004 \\
(-0.33)\end{array}$ & & & & $\begin{array}{l}0.005 \\
(0.39)\end{array}$ & & & \\
\hline $\begin{array}{c}\text { OD } * \text { Purs } \\
\text { from L }\end{array}$ & & $\begin{array}{l}0.015 \\
(0.89) \\
\end{array}$ & $\begin{array}{l}0.011 \\
(0.71) \\
\end{array}$ & $\begin{array}{l}-0.043 \\
(-1.47) \\
\end{array}$ & & $\begin{array}{l}0.006 \\
(0.39) \\
\end{array}$ & $\begin{array}{l}0.003 \\
(0.16) \\
\end{array}$ & $\begin{array}{l}-0.007 \\
(-0.25)\end{array}$ \\
\hline $\begin{array}{c}\text { OD } * \text { Purs } \\
\text { from } \mathrm{H}\end{array}$ & & $\begin{array}{l}-0.006 \\
(-0.28) \\
\end{array}$ & $\begin{array}{l}-0.025 \\
(-1.54)\end{array}$ & $\begin{array}{l}0.006 \\
(0.46) \\
\end{array}$ & & $\begin{array}{l}0.009 \\
(0.49) \\
\end{array}$ & $\begin{array}{l}0.002 \\
(0.12) \\
\end{array}$ & $\begin{array}{l}0.013 \\
(0.93) \\
\end{array}$ \\
\hline Firm size & $\begin{array}{l}0.001 \\
(1.23) \\
\end{array}$ & $\begin{array}{c}0.001 \\
(1.17) \\
\end{array}$ & $\begin{array}{l}0.001 \\
(1.24) \\
\end{array}$ & $\begin{array}{l}0.001 \\
(1.31) \\
\end{array}$ & $\begin{array}{l}-0.000 \\
(-0.08) \\
\end{array}$ & $\begin{array}{l}-0.000 \\
(-0.15) \\
\end{array}$ & $\begin{array}{l}-0.000 \\
(-0.09) \\
\end{array}$ & $\begin{array}{l}-0.000 \\
(-0.01) \\
\end{array}$ \\
\hline Leverage & $\begin{array}{c}-0.116 * * * \\
(-21.41) \\
\end{array}$ & $\begin{array}{c}-0.116 * * * \\
(-21.43)\end{array}$ & $\begin{array}{c}-0.116 * * * \\
(-21.39) \\
\end{array}$ & $\begin{array}{c}-0.116 * * * \\
(-21.37)\end{array}$ & $\begin{array}{c}-0.115 * * * \\
(-21.12)\end{array}$ & $\begin{array}{c}-0.115 * * * \\
(-21.12)\end{array}$ & $\begin{array}{c}-0.115 * * * \\
(-21.12) \\
\end{array}$ & $\begin{array}{c}-0.115 * * * \\
(-21.11)\end{array}$ \\
\hline Public dummy & $\begin{array}{l}-0.007 \\
(-1.55) \\
\end{array}$ & $\begin{array}{l}-0.006 \\
(-1.49) \\
\end{array}$ & $\begin{array}{l}-0.006 \\
(-1.50) \\
\end{array}$ & $\begin{array}{l}-0.006 \\
(-1.50) \\
\end{array}$ & $\begin{array}{c}-0.011 * * * \\
(-2.56) \\
\end{array}$ & $\begin{array}{c}-0.011 * * \\
(-2.50)\end{array}$ & $\begin{array}{c}-0.011 * * * \\
(-2.56)\end{array}$ & $\begin{array}{c}-0.011 * * \\
(-2.54)\end{array}$ \\
\hline Constant & $\begin{array}{l}-0.016 \\
(-0.50)\end{array}$ & $\begin{array}{l}-0.013 \\
(-0.42)\end{array}$ & $\begin{array}{l}-0.017 \\
(-0.53)\end{array}$ & $\begin{array}{l}-0.017 \\
(-0.54)\end{array}$ & $\begin{array}{c}0.062^{*} \\
(1.89) \\
\end{array}$ & $\begin{array}{c}0.065 * * \\
(1.98)\end{array}$ & $\begin{array}{c}0.063 * \\
(1.91) \\
\end{array}$ & $\begin{array}{c}0.061 * \\
(1.87)\end{array}$ \\
\hline YD, ID, GD & Yes & Yes & Yes & Yes & Yes & Yes & Yes & Yes \\
\hline Observations & 6,732 & 6,732 & 6,732 & 6,732 & 6,732 & 6,732 & 6,732 & 6,732 \\
\hline F Value & 15.70 & 15.38 & 15.35 & 15.43 & 15.66 & 15.32 & 15.28 & 15.37 \\
\hline Adj R-Sq & 0.15 & 0.15 & 0.15 & 0.15 & 0.15 & 0.15 & 0.15 & 0.15 \\
\hline
\end{tabular}

\section{Conclusion}

This paper has attempted to determine whether intragroup transactions are used for controlling a family's wealth and whether the transactions among affiliated firms in large family business groups affect firm performance.

First, it finds that intragroup transactions' impacts on firm profitability differ according to the firm's ownership structure (ultimate ownership and position) that are measured with the ownership data of all group firms, including unlisted firms. The test results show that the transactions of firms with large or direct family ownership improve their performance while the other affiliated firms' intragroup transactions worsen their performance. Eventually, they indicate that tunneling could happen in family business groups through intragroup transactions.

However, it is not obvious that the family-owned firms gain from transactions with the affiliated firms that lose from the transactions. Therefore, this paper investigates which affiliates the firms gain from the intragroup transactions. For this analysis, transaction counterparties are divided into two groups using three variables: ultimate ownership, position, and centrality. We discover that firms with large or direct family ownership benefit from intragroup transactions with firms with low ultimate ownership, high position, or high centrality. These results support the prior studies showing that the wealth of firms in which controlling families have less ownership is transferred to firms in which the family has more. Further the results show that central firms are also used for tunneling. This paper also performs the tests on valuation using publicly listed firms and finds that the family-owned listed firms have a higher valuation when they have transactions with the firms where controlling families have low cash flow rights. 


\section{References}

[1] H. Almeida, S. Y. Park, M. G. Subrahmanyam, and D. Wolfenzon, "The structure and formation of business groups: Evidence from Korean chaebols,” Journal of Financial Economics, 99, (2011)

[2] K. H. Bae, J. K. Kang, and J. M. Kim, "Tunneling or value-added? Evidence from mergers by Korean business groups," The Journal of Finance, 57, (2002)

[3] M. Bertrand, P. Mehta, and S. Mullainathan, "Ferreting out tunneling: An application to Indian business groups," The Quarterly Journal of Economics, 117, (2002)

[4] S. J. Chang, "Ownership structure, expropriation, and performance of group-affiliated companies in Korea," Academy of Management Journal, 46, (2003)

[5] S. Claessens, S. Djankov, J. P. H. Fan, and L. H. P. Lang, "Disentangling the incentive and entrenchment effects of large shareholdings," The Journal of Finance, 57, (2002)

[6] M. Faccio, L. H. P. Lang, and L. Young, "Dividends and expropriation," American Economic Review, 91, (2001)

[7] S. Johnson, R. L. Porta, F. Lopez-de-Silanes, and A. Shleifer, "Tunneling," the American Economic Review, 90, (2000)

[8] S. W. Lee and B. G. Chun, "Control-ownership disparity and business performance: A study of Korean firms," Asian Economic Journal, 28, (2014) 
Tunneling through Intragroup Transactions: Evidence from Korean Chaebols

This page is empty by intentions. 